\title{
LES DIAMÈTRES DU SOLEIL DANS LA CONNAISSANCE DES TEMPS DEPUIS 1795
}

\author{
MICHEL TOULMONDE \\ Observatoire de Paris (DANOF)
}

Pour calculer les éphémérides nécessaires à l'observation du Soleil, publiées dans la Connaissance des Temps (CdT), les astronomes du Bureau des longitudes ont utilisé depuis 200 ans différentes valeurs de son diamètre: à l'apogée ou à la distance moyenne de la Terre. Ces données, provenant des meilleures mesures les plus récentes, ont évolué avec la précision des techniques instrumentales.

\section{Le Soleil dans la "Connaissance des Temps" au 19e siècle}

En France, le 19e siècle commence pendant l'an IX de la République "une et indivisible": à la fin du 18e siècle, le calendrier républicain devint d'usage officiel pendant un peu plus de 12 ans, du 6 octobre 1793 (15 vendémiaire an II) au 31 décembre 1805 (10 nivôse an XIV). L'Académie des Sciences est supprimée par la Convention (août 1793) et c'est la Commission temporaire des Poids et Mesures qui publie la CdT pour 1795, "Connoissance des Temps, à l'usage des Navigateurs et des Astronomes pour l'année 1795. Du 12 Nivose de l'an 3 au 10 nivose de l'an \& de l'Ére Républicaine."

Le demi-diamètre solaire y est calculé à partir de la valeur de référence provenant de la mesure du diamètre apogée $\left(31^{\prime} 31^{\prime \prime}\right)$ par Lalande en 1764 [1]. Lalande, chargé des calculs, l'indique de deux façons: dans les pages mensuelles d'abord, tous les 6 jours (les $1,7,13,19$ et 25 du mois), puis à la fin de ces tables mensuelles, les 1 et 16 de chaque mois républicain. Les tableaux mensuels d'éphémérides donnent également la demi-durée de passage du Soleil au méridien (à $0,1 \mathrm{~s}$ près). Comme les mois républicains ont tous la même durée ( 30 jours), c'est la première fois que ces informations sont indiquées dans des tables à des dates équidistantes sur une année.

En 1795, la Convention crée le Bureau des longitudes ( 7 messidor an 3 ou 25 juin 1795 "vieux style"), lequel est alors chargé de rédiger la $\mathrm{CdT}$ 
"plusieurs années à l'avance" (comme le fait le Board of Longitudes anglais) ainsi qu'un "Annuaire propre à régler ceux de toute la République". Le premier volume de la CdT publié par le Bureau des longitudes est celui pour l'an IV (1795-1796), édité en septembre 1795. Concernant le diamètre solaire, il est indiqué (p. 178):

"Le diamètre du Soleil est calculé de 6 en 6 jours sur les tables de Lalande à partir de la valeur apogée de 31'31" observée en 1764 avec un héliomètre de 18 pieds. A cause de l'irradiation, il faut diminuer de $6^{\prime \prime}$ les diamètres que l'on trouve ici dans les tables."

Le premier volume de l'Annuaire est celui de l'an V (1796-1797): “Annuaire de la République française présenté au Corps Législatif par le Bureau des longitudes, pour l'année $V$ de l'Ère française."

Ce petit livre de 40 pages $(8 \mathrm{~cm} \times 10 \mathrm{~cm})$, version pratique et très simplifiée de la CdT, donne entre autres le diamètre du Soleil uniquement le 1 et le 16 de chaque mois républicain; les valeurs proviennent également de la mesure du diamètre apogée $\left(31^{\prime} 31^{\prime \prime}\right)$ par Lalande en 1764 .

L'Annuaire pour l'an IX (1800-1801) donne le diamètre du Soleil "en tems" le 1 et le 16 de chaque mois. Les valeurs des durées de passage du Soleil au méridien indiquées dans l'Annuaire pour l'an XII (1803-1804) sont manifestement incorrectes (ce qui n'est pas le cas dans la CdT): elles décrivent une évolution sinusoïdale annuelle de cette durée ! Les valeurs pour l'an XIII, identiques à celles pour l'an XII, présentent le même défaut, corrigé dans l'Annuaire pour l'an XIV (1805-1806). Celui pour 1808 donne les demi-diamètres du Soleil "en tems", le 1 et le 16 de chaque mois grégorien, le calendrier républicain ayant cessé d'être officiel le 31 décembre 1805.

La présentation et le contenu de la CdT vont continuer d'évoluer suivant les rédacteurs et les éditions. En ce qui concerne le Soleil, les éphémérides du diamètre (ou parfois du demi-diamètre) sont regroupées sur deux pages, à partir de l'édition pour 1838 , et données de façon continue tous les 5 jours, depuis le 0 janvier, ou le 1 janvier selon les années. La valeur de référence reste le diamètre apogée mesuré par Lalande en 1764, correspondant à un demi-diamètre à la distance moyenne $R_{1}=961,4^{\prime \prime}$. Parfois, il est indiqué que cette valeur provient des tables de 1806 calculées par Delambre [2] lequel avait repris la valeur mesurée par Lalande.

En 1876 , la valeur de référence devient $16^{\prime} 1,82^{\prime \prime}$ (ou $R_{1}=961,82^{\prime \prime}$ ), provenant d'observations effectuées à Greenwich de 1836 à 1847 sous la direction de G.-B. Airy [3]. Cette valeur est conservée jusqu'à l'édition pour 1895 (publiée en août 1892) dont l'avertissement indique (page III): "Le demi-diamètre du Soleil à sa distance moyenne de la Terre a été adoptée $=16^{\prime} 1^{\prime \prime}, 82$ ainsi qu'il résulte des observations faites à l'Observatoire de Greenwich, de 1836 à 1847, et sa parallaxe $=8^{\prime \prime}, 86$. Pour le calcul des éclipses et des occultations, nous adoptons pour valeur du demi-diamètre 


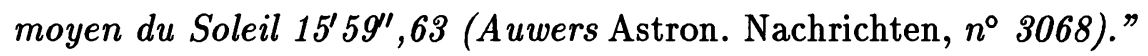

Voici donc 100 ans, cette année, qu'apparaît pour la première fois dans la CdT la valeur établie par Auwers [4] en 1891. En ce qui concerne le diamètre solaire, la $\mathrm{CdT}$ pour 1895 donne jour par jour à midi moyen la durée de passage du demi-diamètre (en temps sidéral et en temps moyen, en $0,01 \mathrm{~s}$ ), le demi-diamètre (en $0,01^{\prime \prime}$ ), et le logarithme du rayon vecteur avec 7 décimales. Les diamètres sont calculés à partir de la valeur $R_{1}=961,82^{\prime \prime}$ en excès de $2,19^{\prime \prime}$ sur la valeur d'Auwers $\left(959,63^{\prime \prime}\right)$.

\section{Le Soleil dans la "Connaissance des Temps" au 20e siècle}

En 1911 se tient à l'Observatoire de Paris le Congrès International des Éphémérides astronomiques (23-26 octobre 1911). Concernant les dimensions du Soleil, la deuxième résolution indique: "De même pour [unifier] le

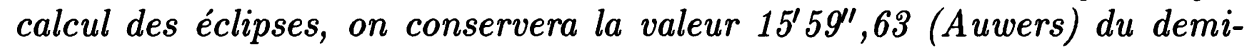
diamètre apparent $d u$ Soleil qui est actuellement employée par toutes les éphémérides".

Cette résolution est appliquée dès la publication en mars 1912 de la CdT pour 1914; on y lit (p. 12): "Le demi-diamètre vaut 16' 1",18 (d'après

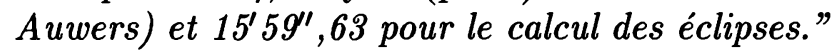

La CdT pour 1915 précise: "Le demi-diamètre $d u$ Soleil est $s=15^{\prime} 59^{\prime \prime}, 63$ (Auwers), valeur adoptée pour le calcul des éclipses par la Conférence internationale des Éphémérides astronomiques. Pour le calcul des éphémérides, la Connaissance des Temps prend, d'après Auwers en tenant compte de l'irradiation $s=16^{\prime} 1^{\prime \prime}, 18$.

Depuis, ces valeurs n'ont pas été modifiées et, pour chaque année jusqu'à 1979, la CdT donne, jour par jour à midi vrai de Greenwich, la durée de passage du demi-diamètre (en temps sidéral) et le demi-diamètre (en ' et ").

Mais depuis 1980, la CdT ne donne plus de table du diamètre du Soleil : la "Nouvelle série" fournit des coefficients pour calculer les coordonnées sous forme de développements en polynômes de Tchebychev. La distance $\mathrm{du}$ Soleil est ainsi calculable avec une précision inférieure à $1 \times 10^{-6} \mathrm{UA}$.

Le système des constantes conventionnellement utilisées dans le calcul des éphémérides astronomiques, modifié en 1976 par la 16e assemblée générale de l'UAI (Grenoble), est en vigueur depuis 1984. Parmi les valeurs "recommandées" figure le rayon équatorial du Soleil qui doit être pris égal à $696000 \mathrm{~km}$. Le Supplément à la CdT pour les "Satellites galiléens de Jupiter, phénomènes et configurations" indique cependant dans chacune de ses éditions annuelles: "Le Soleil est une sphère de rayon $695980 \mathrm{~km}$."

L'Annuaire du Bureau des longitudes, utilise les mêmes valeurs de référence. Pour la table des diamètres solaires, jusqu'en 1911, la référence est 
961,82" (Airy 1855). Il est indiqué que la distance moyenne du Soleil est de 149501 milliers de kilomètres. Dans l'édition pour 1912, il est écrit (p. 246): "Le diamètre du Soleil est de 32'02",36 $\left[R_{1}=961,18^{\prime \prime}\right]$ mais cette valeur est un peu trop grande à cause de l'irradiation, et le diamètre réel doit être réduit à $31^{\prime} 5 g^{\prime \prime}, 26\left[R_{1}=959,63^{\prime \prime}\right]$."

L'information reste la même de 1912 à 1951, et dans l'édition pour 1952 , seule la valeur $31^{\prime} 59,26^{\prime \prime}$ est citée, et ce jusqu'en 1974. Depuis 1975, le diamètre de référence n'est plus indiqué dans l'Annuaire.

Pour la table des diamètres du Soleil dans l'Annuaire, les valeurs sont indiquées avec une périodicité de 15 jours (de 1796 à 1967), puis de 10 jours de 1968 à 1976, et enfin de 8 jours depuis 1977. La table donne le demidiamétre $(\mathrm{D} / 2)$ à $12 \mathrm{~h}$ UT et la distance du Soleil $(\mathrm{d})$ à $0 \mathrm{~h} \mathrm{UT}$. On ne peut pas vérifier directement la constance du produit $K_{1}=d . D / 2$, les données n'étant pas établies pour le même instant; une interpolation est nécessaire, et on constate effectivement que $K_{1}$ vaut $961,18^{\prime \prime}$ correspondant à la somme $959,63^{\prime \prime}+1,55^{\prime \prime}$ en tenant compte de l'irradiation pour les observations du disque solaire.

Il est à noter que cette valeur $1,55^{\prime \prime}$ utilisée pour l'irradiation est toujours référencée comme étant due à Auwers; toutefois, son article [4] indique l'écart $3,14^{\prime \prime}$ (double de 1, 57") entre la valeur du diamètre apparent utilisée par le Berliner Jahrbuch et celle qu'il déduit des mesures qu'il a effectuées. L'écart cité n'est donc pas de $3,10^{\prime \prime}$ (double de 1,55"). Peut-être s'agit-il d'un arrondi qui se perpétue?

Deux valeurs du demi-diamètre solaire sont utilisées simultanément depuis un siècle: l'une pour le calcul des éclipses $\left(959,63^{\prime \prime}\right)$, l'autre pour les observations physiques du Soleil $\left(961,18^{\prime \prime}\right)$. L'évolution de ces valeurs de référence traduit l'amélioration des techniques d'observation, ainsi que les grandes difficultés à déterminer rigoureusement le bord solaire, dont les limites sont souvent vues de façons différentes selon les observateurs [5].

\section{Références}

Lalande J.J.(de), (1764), Astronomie.

Bureau des longitudes, 1806, Tables astronomiques.

Airy G.B., 1855, Observations Made at the Royal Observatory Greenwich in the year 1853, London.

Auwers A., 1891, "Der Sonnendurchmesser und der Venusdurchmesser nach den Beobachtungen an den Heliometern der deutschen Venus-Expeditionen.", Astron. Nachrichten, 3068(128), 361.

Toulmonde M., 1995, Étude comparative de diamètres solaires observés à partir d'instruments astrométriques, Thèse de doctorat, Observatoire de Paris. 